\title{
ARTICLE
}

Received 18 Sep 2013 | Accepted 12 Feb 2014 | Published 17 Mar $2014 \quad$ DOl: 10.1038/ncomms4432

\section{Designing a robustly metallic noncenstrosymmetric ruthenate oxide with large thermopower anisotropy}

Danilo Puggioni ${ }^{1} \&$ James M. Rondinelli ${ }^{1}$

The existence of $\sim 30$ noncentrosymmetric metals (NCSM) suggests a contraindication between crystal structures without inversion symmetry and metallic behaviour. Those containing oxygen are especially scarce. Here we propose and demonstrate a design framework to remedy this property disparity and accelerate NCSM oxide discovery. The primary ingredient relies on the removal of inversion symmetry through displacements of atoms whose electronic degrees of freedom are decoupled from the states at the Fermi level. Density functional theory calculations validate this crystal-chemistry strategy, and we predict a new polar ruthenate exhibiting robust metallicity. We demonstrate that the electronic structure is unaffected by the inclusion of spin-orbit interactions, and that cation-ordered $\mathrm{SrCaRu}_{2} \mathrm{O}_{6}$ exhibits a large thermopower anisotropy $\left(\left|\Delta \mathrm{S}_{\perp}\right| \sim 6.3 \mu \mathrm{VK}-1\right.$ at $\left.300 \mathrm{~K}\right)$ derived from its polar structure. Our findings provide chemical and structural selection guidelines to aid in the search of NCSM with enhanced thermopower anisotropy.

\footnotetext{
${ }^{1}$ Department of Materials Science \& Engineering, Drexel University, Philadelphia, Pennsylvania 19104, USA. Correspondence and requests for materials should be addressed to J.M.R. (email: jrondinelli@coe.drexel.edu).
} 
T he metallic features in materials, which provide lowresistance channels for electrical conduction, lead to effective screening of local electric dipole moments ${ }^{1}$. Itinerant electrons disfavour both their formation and cooperative ordering ${ }^{2}$. In spite of the incompatibility between acentricity and metallicity, metallic materials that break the spatial parity operation mapping $(x, y, z) \rightarrow(\bar{x}, \bar{y}, \bar{z})$ were originally discussed in the 1960 s by Matthias and then, later more rigorously by Anderson and Blount ${ }^{3}$. The first experimental identification ${ }^{4}$ of a candidate polar metal, the binary intermetallic $\mathrm{V}_{2} \mathrm{Hf}$, was made a decade later. Since then, more noncentrosymmetric metals (NCSM) have been identified (Fig. 1) and found to exhibit unconventional optical responses ${ }^{5,6}$, magnetoelectricity ${ }^{7,8}$ and superconductivity ${ }^{9-11}$. Yet, they remain challenging to discover. The principal NCSM material classes are binary and ternary intermetallics and silicides. Intriguingly, few NCSM are oxides with the notable exceptions ${ }^{12-15}$ of a layered cuprate $\left(\mathrm{Y}_{1-x} \mathrm{Ca}_{x} \mathrm{Sr}_{2} \mathrm{GaCu}_{2} \mathrm{O}_{7 \pm y}\right)$, a geometrically frustrated pyrochlore $\left(\mathrm{Cd}_{2} \mathrm{Re}_{2} \mathrm{O}_{7}\right)$, and recently $\mathrm{LiOsO}_{3}$ with a structural transition similar to ferroelectric $\mathrm{LiNbO}_{3}$. (Degenerately doped dielectrics like $\mathrm{BaTiO}_{3-\delta}$ (ref. 16) and $\mathrm{ZnO}: \mathrm{Al}^{\mathrm{l}}$, although exhibiting metallic resistivity, are not intrinsically metallic and NCS-their conductivity is high because of deviations from ideal stoichiometry.) Figure 1 suggests that NCSM tend to require cations with large atomic masses, but this is not always the case, for example, $\mathrm{Mg}_{2} \mathrm{Al}_{3}$. The absence of reliable crystal-chemistry guidelines and the limited understanding of the microscopic origin of inversion symmetrylifting displacements in metals poses a serious challenge for their discovery: how does one explain, let alone design, cooperative acentric atomic displacements in crystalline oxide conductors?

In this work, we present a microscopic understanding for NCSM, leading to a design principle that we use in the theoretical prediction of a polar NCSM transition metal (M) oxide. We formulate a two-step material selection process based on a weak electron-lattice coupling principle that considers the symmetry and atomic species participating in the inversion-lifting lattice modes, and the susceptibility of the cations' electronic configurations to a metallic state. We show that two $k \neq 0$ lattice modes describing rotations of $\mathrm{MO}_{6}$ octahedra induce polar A-cation displacements in the layered and cation-ordered $(\mathrm{Sr}, \mathrm{Ca}) \mathrm{Ru}_{2} \mathrm{O}_{6}$ perovskite, for which experimental solid solutions ${ }^{18}$ are metallic.
Because the microscopic mechanism is independent of the details of the states at the Fermi level, unlike conventional dipolar interactions that are essential to stabilizing acentric displacements of second-order Jahn-Teller cations in dielectrics ${ }^{19}$, the design strategy is readily transferable to other transition metal chemistries. We show that spin-orbit interactions (SOI) do not alter the electronic ground state, and that the unusual coexistence of a polar axis and metallicity produces a novel correlated material possessing a highly anisotropic thermopower response $\left(\left|\Delta S_{\perp}\right| \sim 6.3 \mu \mathrm{V} \mathrm{K}^{-1}\right.$ at $300 \mathrm{~K}$ ) with nearly isotropic electrical conductivity.

\section{Results}

Design of a metallic oxide without inversion. Our design strategy originates from Anderson's work ${ }^{3}$ on 'ferroelectric metals,' where he writes that "while free electrons screen out the electric field [in materials] completely, they do not interact very strongly with the transverse optical phonons and the Lorentz local fields [that] lead to ferroelectricity, since umklapp processes are forbidden as $k \rightarrow 0$." We recast this observation into an operational principle that states: the existence of any NCSM relies on weak coupling between the electrons at the Fermi level, and the (soft) phonon(s) responsible for removing inversion symmetry. An essential, implicit, materials constraint is that the low-energy electronic structure derives from an electron count giving partial band occupation, which may be obtained by judicious selection of the cation chemistries.

We identify three displacive routes fulfilling the weak-coupling hypothesis in solid-state systems, specified by the symmetry behaviour of the lattice phonons that would remove inversion symmetry in a centrosymmetric metal and yield a NCS crystal structure (Table 1). It should be noted that an order-disorder mechanism could also be operative; the classification of such symmetry breaking is consistent with our scheme; the caveat being that the soft phonon is replaced by an order parameter that describes atomic site occupancy. In either case the same set of irreducible representations may be used.

The third approach, which we focus on here, resembles a condition that supports a novel 'improper' mechanism ${ }^{20,21}$ for ferroelectricity that has been exploited to design artificial polar oxides with sizeable electric polarizations by heterostructuring non-polar dielectrics. Note that throughout we choose to refer to

\section{OPTICAL ACTIVITY (CIRCULAR DICHORISM)}

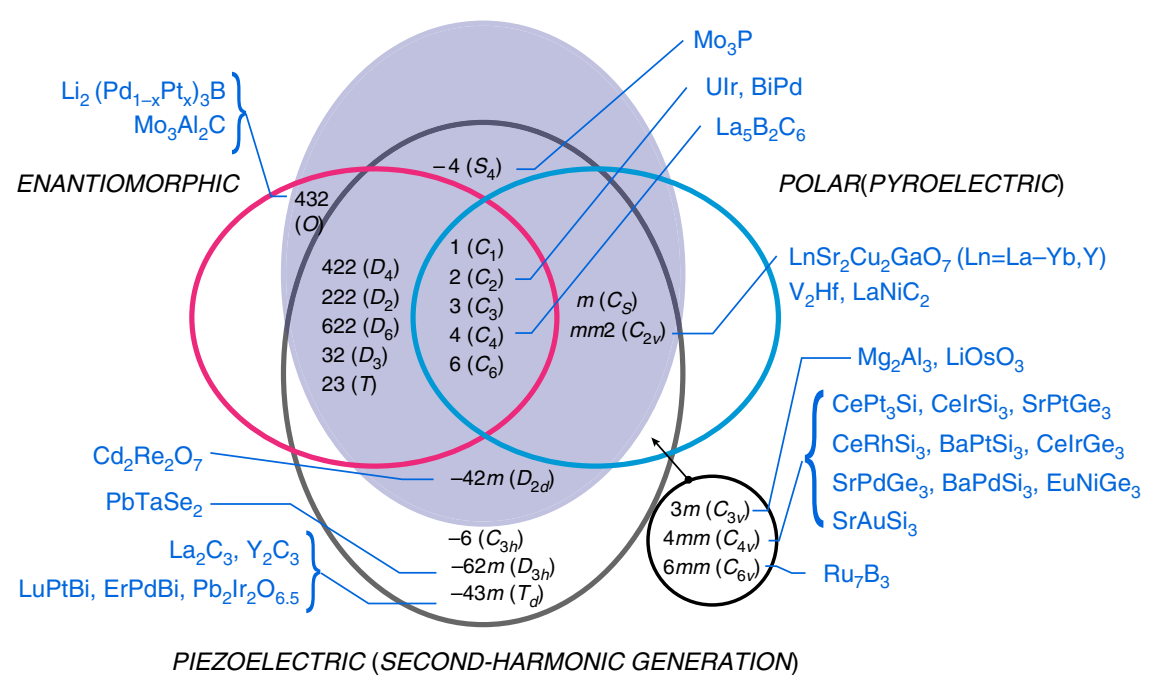

Figure 1 | Classification of noncentrosymmetric metals. Known compounds differentiated by crystal class following the NCS classification scheme introduced in Halasyamani and Poeppelmeier ${ }^{65}$ include $4 d$ and $5 d$ transition metal intermetallics, carbides or silicides. Oxide compounds are scarce. 
Table 1 | Routes to lift inversion symmetry in metallic compounds.

\begin{tabular}{lll} 
Inversion-lifting method & $\begin{array}{l}\text { Mode } \\
\text { requirements }\end{array}$ & Description \\
\hline (1) Compositional order & None & $\begin{array}{l}\text { Achieved by decorating one or more interleaved lattices with multiple cations, e.g., as realized in half- } \\
\text { Heulser alloys or tri-colour, ./ABC/ABC/., superlattices. }\end{array}$ \\
$\begin{array}{l}\text { (2) Packing of acentric } \\
\text { polyhedra }\end{array}$ & $\begin{array}{l}\text { Realized by the alignment of acentric metal-oxygen polyhedral units arising either from out-of-centre } \\
\text { mode } k=0 \text { or } k \neq 0\end{array}$ & $\begin{array}{l}\text { Retal distortions or because they intrinsically lack inversion }\left(\mathrm{MO}_{4} \text { tetrahedron), and contribute few }\right. \\
\text { (if any) states to or near the Fermi level. }\end{array}$ \\
$\begin{array}{l}\text { (3) Geometric-induced } \\
\text { displacements }\end{array}$ & $\begin{array}{l}\geq 2, k \neq 0 \\
\text { Obtained by anharmonic coupling of two or more centric lattice modes, which cooperatively remove } \\
\text { inversion and produce cation displacements that do not gap the electronic structure. Accessible in } \\
\text { some, effectively, two-dimensional compounds, for example, naturally layered structures or } 3 \mathrm{D} \text { systems } \\
\text { with bi-colour ordering. }\end{array}$
\end{tabular}

The scheme is formulated by classifying the symmetry requirements of the lattice mode instabilities of a centrosymmetric (reference) phase, which would drive a thermodynamic transition to a noncentrosymmetric crystal structure. Although compositional ordering may be an obvious route to lift inversion symmetry, we recognize that experimentally in practice it may be challenging to achieve it, especially in oxide materials owing to bond-coordination requirements; therefore, although the route is simple, it is by no means straightforward. The design methodology demonstrated here implements method (3). Note that all materials are expected to show some interesting physical properties enabled by an acentric crystal structure with itinerant electrons, independent of the mechanism leading to the mutual coexistence of the prerequisite atomic and electronic structure.

these materials as NCS metals rather than 'ferrroelectric metals' as articulated by Anderson and recently others to eliminate misconceptions about the presence of switchable spontaneous electric polarizations in metals, and more accurately describe the crystallographic-electronic function of the materials. In this way, one can generally and completely classify all metals without inversion symmetry and not solely those of the polar subset of NCS structures (Fig. 1).

Within this framework, the design of a NCSM oxide using geometric-induced displacements requires an oxide class exhibiting coupled zone-boundary phonons that lift inversion symmetry. Here we choose orthorhombic $\mathrm{AMO}_{3}$ perovskites with cornerconnected $\mathrm{MO}_{6}$ octahedra that are rotated about each Cartesian axis; the 'tilt' pattern is obtained and described by two phonons with $k=(1 / 2,1 / 2,0)$ and $(1 / 2,1 / 2,1 / 2)$ wavevectors relative to the cubic aristotype ${ }^{22}$. The $a^{+} b^{-} c^{-} \mathrm{MO}_{6}$ rotation pattern in the presence of layered A-cation order along a [001] direction, for example, accessible through synthetic bulk chemistry routes ${ }^{23,24}$ or heteroepitaxial thin-film growth methods ${ }^{25}$, is sufficient to produce a polar structure ${ }^{26,27}$. The chemical species are selected given the constraints that the rotations of octahedra are necessary-determined by the $\mathrm{A}$ and $\mathrm{M}$ cation size mismatch in perovskites-and that the electronic configuration of the $\mathrm{M}$ cation results in a nonzero density-of-states at the Fermi level $\left(E_{\mathrm{F}}\right)$. These requirements should be satisfied for ordered $(\mathrm{Sr}, \mathrm{Ca}) \mathrm{Ru}_{2} \mathrm{O}_{6}$, since both bulk $\mathrm{SrRuO}_{3}$ and $\mathrm{CaRuO}_{3}$ are experimentally found in the $a^{+} b^{-} c^{-}$Glazer tilt pattern and also have partially occupied low-energy $\mathrm{Ru} 4 d t_{2 g}$ orbitals.

Ground-state structure. Figure 2 depicts the polar $\mathrm{Pmc}_{1}$ ground state crystal structure of $(\mathrm{Sr}, \mathrm{Ca}) \mathrm{Ru}_{2} \mathrm{O}_{6}$ obtained from density functional theory calculations. (See Supplementary Table 1 for the crystallographic data.) The extended $\mathrm{RuO}_{6}$ structure is highly rotated, adopting the targeted orthorhombic tilt pattern with rotations of adjacent octahedra out-of-phase about the [011] direction and in-phase about the layering [100] direction (Fig. 2a). The $\mathrm{Ru}-\mathrm{O}-\mathrm{Ru}$ bond angles are $157.1^{\circ}$ and $145.6^{\circ}$ (perpendicular to the $\mathrm{CaO} / \mathrm{SrO}$ layers) and $150.4^{\circ}$ (within the layers). The stabilization of this rotation pattern is understood on the grounds that the $\pi^{*}$ band, which is nearly full because of the $t_{2 g}^{4}$ configuration, is shifted to lower energy by the electronegative $\mathrm{Ru}^{4+}$ cation ${ }^{28}$.

( $\mathrm{Sr}, \mathrm{Ca}) \mathrm{Ru}_{2} \mathrm{O}_{6}$ exhibits small off-centre Ru distortions (Fig. 2b) and large A-site cation displacements (Fig. 2c,d). Acentric B-site displacements are common to TMs with $d^{0}$-electronic configurations in octahedral coordinations; however, for $d^{n}, n \geq 1$, such polar displacements are largely disfavoured, that is, the energetic gain due to $d \pi-p \pi$ metal-oxygen bonding decreases on filling the $t_{2 g}$ orbital ${ }^{29}$. Nonetheless, we find they occur for $d^{4} \mathrm{Ru}^{4+}$, displacing the metal centre nearly towards an edge $(\sim 0.03 \AA)$, staying in the (110) plane (see Supplementary Table 2). This results in one short, two long and three medium $\mathrm{Ru}-\mathrm{O}$ bonds (Fig. 2b), which are close to the average value found experimentally in the $\mathrm{Sr}_{0.5} \mathrm{Ca}_{0.5} \mathrm{Ru}_{2} \mathrm{O}_{3}$ solid solution ${ }^{30}$. The $\mathrm{Ca}$ and $\mathrm{Sr}$ atoms make large polar displacements $(0.29$ and $0.21 \AA$, respectively) along the [001] and [001] directions (see Supplementary Table 3), resulting in distorted polyhedra (Fig. 2c). We explore these displacements and their effect on the electronic structure in more detail below.

Electronic properties. Figure 3 shows the total and partial densities-of-states (DOS) for the polar NCSM $(\mathrm{Sr}, \mathrm{Ca}) \mathrm{Ru}_{2} \mathrm{O}_{6}$ without (Fig. 3a) and with (Fig. 3b) SOI. The valence band is composed largely of $\mathrm{O} 2 p$ states hybridized with $\mathrm{Ru} 4 d$ states, with the oxygen states predominately found in regions below the $E_{\mathrm{F}}$. Consistent with low-spin $\mathrm{Ru}^{4+}$, the large peaks in the DOS near $E_{\mathrm{F}}$ are caused by the fairly flat $\mathrm{Ru} t_{2 g}$ bands, whereas the strongly hybridized $e_{g}$ orbitals form broader bands in the conduction band beginning near $1.5 \mathrm{eV}$. We find that without SOI, weakly dispersive $t_{2 g}$ bands lead to a large number of states at the Fermi level, that is, $N\left(E_{\mathrm{F}}\right) \sim 2.2 \mathrm{eV}$ per spin per Ru atom indicating robust metallic behaviour (Fig. 3a), despite a $\sim 0.75 \mathrm{eV}$ band gap present in both spin channels far above $E_{\mathrm{F}}(0.5 \mathrm{eV})$. It is also ferromagnetic $\left(0.914 \mu_{\mathrm{B}}\right.$ f.u. $\left.{ }^{-1}\right)$ with $\sim 0.35 \mu_{\mathrm{B}}$ per $\mathrm{Ru}$ atom; the remaining magnetization is distributed among the oxygen ligands due to the itinerant spin-polarized electrons. Using a simple modified Weiss formula ${ }^{31}$, we calculate a Curie temperature $T_{\mathrm{c}}=47 \mathrm{~K}\left(27 \mathrm{~K}\right.$ applying Anderson's rescaling $\left.{ }^{32}\right)$ in good agreement with experimental data ${ }^{18}$ on solid solution $\mathrm{Sr}_{0.5} \mathrm{Ca}_{0.5} \mathrm{RuO}_{3}\left(T_{\mathrm{c}}=57 \mathrm{~K}\right)$.

Since SOI play a significant role in determining the orbital structure in $3 d$ transition metal oxides ${ }^{33,34}$, we calculate the DOS with SOI (Fig. 3b). Although the SOI enable the spin-up and spin-down bands to mix, we find an electronic structure similar to that without the interactions. The Ru $4 d$-band occupation at $E_{\mathrm{F}}$ is weakly modified and the gap in the conduction band is slightly reduced. Nonetheless, the weakly dispersed $t_{2 g}$ states persist, and $(\mathrm{Sr}, \mathrm{Ca}) \mathrm{Ru}_{2} \mathrm{O}_{6}$ remains metallic $\left[N\left(E_{\mathrm{F}}\right) \sim 4.36 \mathrm{eV}\right.$ per $\mathrm{Ru}$ atom] consistent with SOI calculations on bulk $\mathrm{SrRuO}_{3}$ (ref. 35), where spin-orbit coupling also does not greatly alter the electronic structure.

Origin of acentricity. For purposes of evaluating the design approach and the proposed geometric-induced inversion 

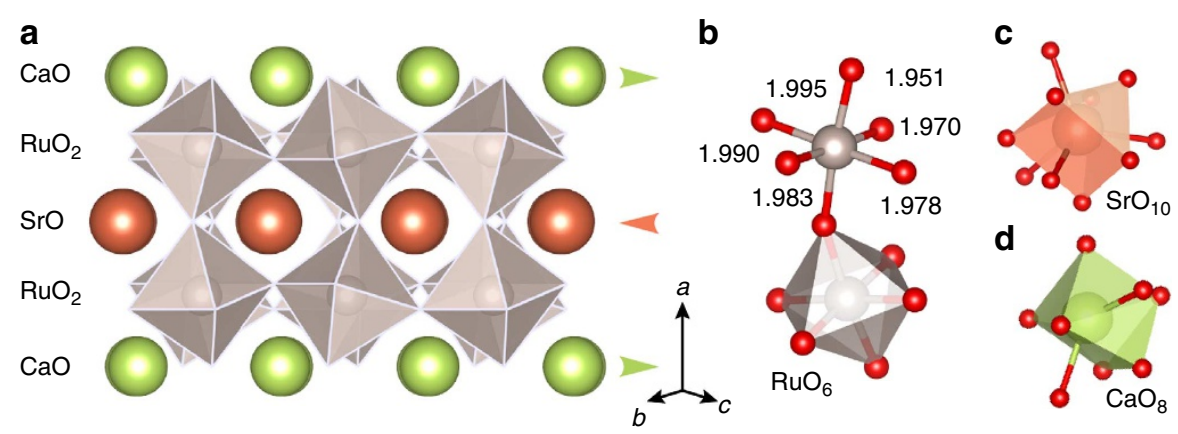

Figure 2 | Ground state structure of the polar noncentrosymmetric metal $\left(\mathbf{S r}, \mathbf{C a}_{\mathbf{2}} \mathbf{R u}_{\mathbf{2}} \mathbf{O}_{\mathbf{6}}\right.$. Crystal structure of cation-ordered $(\mathrm{Sr}, \mathrm{Ca}) \mathrm{Ru}_{2} \mathrm{O}_{6}$ with the $a^{+} b^{-} b^{-} \mathrm{RuO}_{6}$ tilt pattern (a). The off-centre $\mathrm{Ru}^{4+}$ distortions (bond lengths in $\AA$ ) produce local dipoles that cooperatively order along the $c$ direction (b), removing the mirror plane perpendicular to the A-site cation order. The local coordination environment for (c) Sr (tetra-capped trigonal prism) and (d) Ca (bi-capped trigonal prism) reveals that large A-site distortions from the ideal 12-fold coordination occur in opposite directions (arrows), along the polar $\mathrm{c}$ axis.

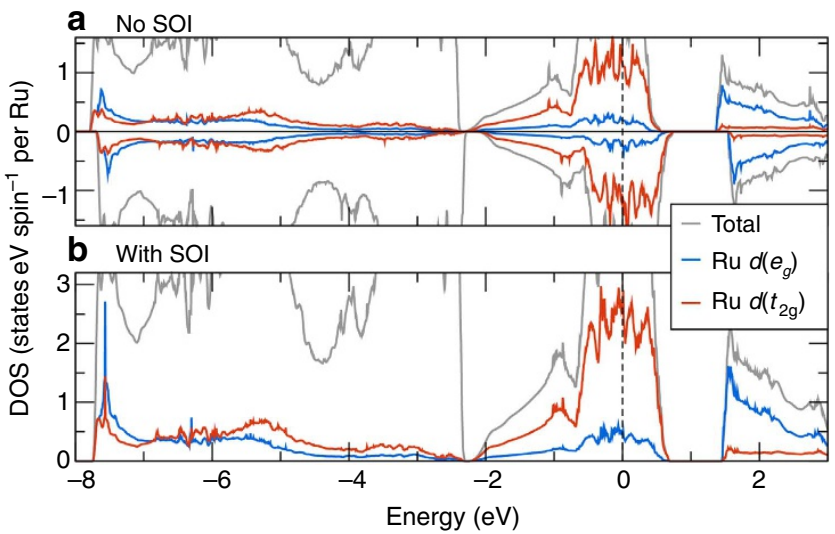

Figure 3 | Electronic structure. The total (black line) and Ru $4 d$ orbital- and spin-resolved densities of states (DOS) of orthorhombic $(\mathrm{Sr}, \mathrm{Ca}) \mathrm{Ru}_{2} \mathrm{O}_{6}$ calculated (a) without spin-orbit interactions (SOI) and (b) with SOI. The Fermi level is at $\mathrm{OeV}$ (dashed line).

symmetry-breaking displacements, we perform a group theoretical analysis ${ }^{36,37}$ of the $P m c 2_{1}$ structure with respect to a fictitious prototypical centrosymmetric phase $(P 4 / \mathrm{mmm}$ symmetry), reducing the polar structure into a set of symmetryadapted modes associated with different irreducible representations of the $P 4 / \mathrm{mmm}$ phase (see Supplementary Fig. 1 and Supplementary Table 4). Note that the hypothetical structure would be related to an experimentally accessible hightemperature phase, assuming that the crystal would not first decompose. The normalized distortion vector is $\xi \simeq 0.32 \Gamma_{5}^{-}$ $+0.54 M_{2}^{+}+0.78 M_{5}^{-}$, where $\Gamma_{5}^{-}$corresponds to a polar $i r$-active mode, and $M_{2}^{+}$and $M_{5}^{-}$describe $k \neq 0$ in-phase and out-of-phase rotation modes, respectively.

The amplitude and energetic contribution of each irrep in the polar structure provide insight into the stability of the NSCM (Fig. 4). Although the zone-centre polar mode transforming as irrep $\Gamma_{5}^{-}$is weakly unstable in the high-symmetry $P 4 / \mathrm{mmm}$ structure (see Supplementary Fig. 2 and Supplementary Table 5), that is, characterized by a negative quadratic energy surface with respect to the increasing amplitude of the polar distortion (Fig. 4a), its energetic contribution to the ground state structure is two orders of magnitude smaller than that obtained from the octahedral rotation modes (Fig. 4b). To show that the geometric $\mathrm{RuO}_{6}$ rotations drive the polar $\mathrm{Sr}$ and $\mathrm{Ca}$ displacements (Fig. 4a, inset), circumventing the contraindication between metallicity and the ir-mode, we map the total energy evolution of
$(\mathrm{Sr}, \mathrm{Ca}) \mathrm{Ru}_{2} \mathrm{O}_{6}$ with increasing amplitude of the ir-polar mode at different fixed values of the $a^{+} b^{-} b^{-}$tilt pattern described by $\left(M_{2}^{+} \oplus M_{5}^{-}\right)$(Fig. 4c). We find that with increasing amplitude of the $a^{+} b^{-} b^{-}$tilt pattern, the negative curvature about the origin vanishes; the energy landscape evolves to a parabola with a single energy minimum, indicating the hardening (stabilization) of the polar phonon mode at finite amplitude induced by the geometric $\mathrm{RuO}_{6}$ octahedral rotations, which fully accounts for the loss of inversion symmetry.

Figure $4 \mathrm{~d}$ shows there are minor changes in the position of the band centre of mass for the principal atoms involved in the polar mode, that is, the $\mathrm{Ca}(4 s 3 d)$ and $\mathrm{Sr}(5 s 4 d)$ states, which are located $\sim 6.2 \mathrm{eV}$ above $E_{\mathrm{F}}$ in the conduction band. The relative change in the number of states at the Fermi level $\left(\Delta N\left(E_{\mathrm{F}}\right)\right.$, Fig. $\left.4 \mathrm{e}\right)$ is also small with respect to the centrosymmetric structure and increasing amplitude of the polar mode, confirming the weak interaction between the inversion symmetry-lifting displacements and the low-energy electronic structure. The conduction bands marginally shift to higher energy $(\sim 0.06 \mathrm{eV})$ and the change in $N\left(E_{\mathrm{F}}\right)$ is $\sim \pm 5 \%$, validating the proposed NCSM design guidelines. Interestingly, the polar mode activates the ferromagnetic ground state observed by the exchange split electronic structure that appears around $\sim 0.25 \AA$ in Fig. $4 \mathrm{e}$, indicative of large spin-phonon coupling ${ }^{38,39}$.

Thermoelectric response. Owing to the orthorhombicity in $(\mathrm{Sr}, \mathrm{Ca}) \mathrm{Ru}_{2} \mathrm{O}_{6}$, the recent interest in thermoelectric properties of perovskite oxide superlattices ${ }^{40}$, and the fact that degenerately doped ferroelectrics with polar axes exhibit unusual thermoelectric properties ${ }^{41-43}$, we evaluate the thermopower anisotropy at $300 \mathrm{~K}$ using Botlzmann transport theory within the constant scattering time approximation.

Figure 5 shows the relevant thermoelectric properties in the chemical potential range varying from -0.25 to $0.25 \mathrm{eV}$. The average thermopower $\vec{S}$, denoted by the broken line in Fig. $5 \mathrm{a}$, ranges from 14 to $-18 \mu \mathrm{V} \mathrm{K}^{-1}$ with two sign changes near $\mu \simeq-0.21$ and $\mu \simeq 0.07 \mathrm{eV}$, indicating a change in the dominant charge carriers from holes to electrons and then from electrons to holes, respectively. We also note that above $\mu \simeq 0.16 \mathrm{eV}$, the thermopower components along the polar $c$ direction dominate the thermal properties.

The conductivity $\sigma$ is found to oscillate in a narrow window over the chemical potential range considered, and all the three components resolved along the crystallographic axes exhibit similar behaviour (Fig. 5b). Above $\mu \simeq 0.05 \mathrm{eV}$, where the Seebeck coefficient along the polar axis is extremized, the conductivity 

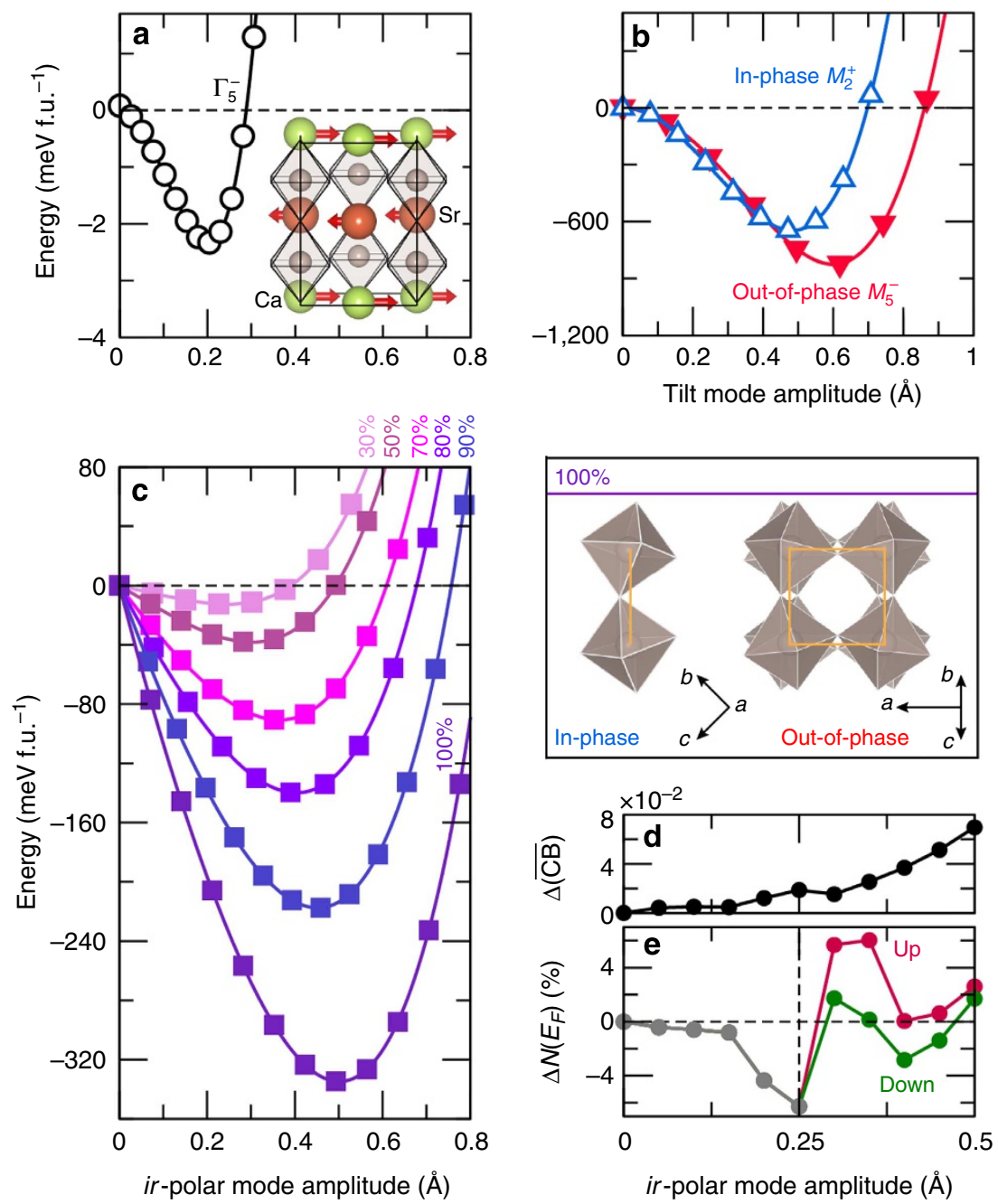

Figure 4 | Electron-lattice interactions on the ground state stability. (a) Polar (ir-active) mode with $\Gamma_{5}^{-}$symmetry. The inset depicts the polar cation displacement pattern; apical oxygen and (small) Ru displacements are omitted for clarity. (b) In-phase and out-of-phase $\mathrm{RuO}_{6}$ rotational modes. (c) Normalized energy gain obtained by increasing amplitude of the polar displacements at fixed percentages of the total $a^{+} b^{-} b^{-}$tilt pattern found in the ground-state structure, that is, described by the combination $M_{2}^{+} \oplus M_{5}^{-}$. The inset depicts the $100 \%$ rotational distortions. (d) Change in the position of the $\mathrm{Sr}$ and $\mathrm{Ca}$ conduction band centre of mass $(\Delta \overline{\mathrm{CB}})$ and $(\mathbf{e})$ variation in the number of states at the Fermi level $\left[\Delta N\left(E_{\mathrm{F}}\right)\right]$ normalized to the value without the polar distortion. The ferromagnetic state is stable for polar displacements larger than $\sim 0.25 \AA$.

along the $c$ axis $\left(\sigma_{c}\right)$ begins to exceed that along the other directions.

Figure $5 c$ shows the total and crystal axis-decomposed power factor $\mathrm{PF}=\sigma S^{2}$. The total power factor exhibits a pronounced local maximum, $\mathrm{PF} \simeq 0.7 \mu \mathrm{W} \mathrm{K}^{-2} \mathrm{~cm}^{-1}$, near $\mu=-0.12 \mathrm{eV}$ (hole doping $h=3 \times 10^{21} \mathrm{~cm}^{-3}$ ), and two other less pronounced local maxima, $\mathrm{PF} \simeq 0.35 \mu \mathrm{W} \mathrm{K} \mathrm{K}^{-2} \mathrm{~cm}^{-1}$ at $\mu \sim 0.05 \mathrm{eV}$ and $\mu \sim 0.18 \mathrm{eV}$, which are negligible when compared with thermoelectric materials found in applications $\left(20-50 \mu \mathrm{W} \mathrm{K}^{-2} \mathrm{~cm}^{-1}\right)^{44}$. It is interesting, however, that the maximum at $\mu \sim 0.18 \mathrm{eV}$ (electron doping of $n=6 \times 10^{21} \mathrm{~cm}^{-3}$ ) is in the region where the Seebeck coefficient and the conductivity along the polar axis both dominate the transport behaviour along the other directions.

To show that this anisotropy arises from the polar displacements and not the orthorhombic lattice symmetry of $(\mathrm{Sr}, \mathrm{Ca}) \mathrm{Ru}_{2} \mathrm{O}_{6}$, we calculate the thermopower anisotropy in the $a b$ plane as $\Delta S_{\|}=S_{a}-S_{b}$ (Fig. $5 \mathrm{~d}$ ), and the thermopower anisotropy along the polar $c$ axis as $\Delta S_{\perp}=S_{c}-\frac{1}{2}\left(S_{a}+S_{b}\right)$ (Fig. 5f), for the equilibrium $P m c 2_{1}$ structure and for a hypothetical structure with the polar atomic displacements described by irrep $\Gamma_{5}^{-}$fully removed.
When we remove the polar displacements, we find that $\Delta S_{\|}$is modified for all chemical potentials examined, except at $0 \mathrm{eV}$. The difference between the two cases $\Delta S_{\|}$ranges between $\pm 15 \mu \mathrm{VK}^{-1}$ (Fig. 5e). The thermopower anisotropy along the polar $c$ axis, $\Delta S_{\perp}$, behaves as $\Delta S_{\|}$, except for a sign change at $0 \mathrm{eV}$ : The difference in the $c$ axis anisotropy, $\delta S_{\perp}$, also ranges between $\pm 15 \mu \mathrm{VK}^{-1}$ for the two structures (Fig. $5 \mathrm{~g}$ ). Consistent with our design guidelines, at $\mu=0 \mathrm{eV}$, the change in the thermopower anisotropy is essentially zero, indicating weak coupling between the states at the Fermi level and the polar atomic displacements that lift inversion symmetry. The role of the polar distortions becomes even more evident by analysing the anisotropy change in the $b c$ plane (see Supplementary Fig. 3) where the lattice parameters are identical. The removal of polar displacements is found to produce a shift relative to the chemical potential below $\mu=0.18 \mathrm{eV}$. Above this value, we find that the anisotropy in the $b c$ plane is small in the structure without the polar distortions, whereas it is maximized in the equilibrium structure. Such behaviour is also observed in Fig. $5 \mathrm{f}$ over the same electron-doped region. The magnitude of the anisotropy, $\left|\delta S_{\perp}\right|$, in the ground 


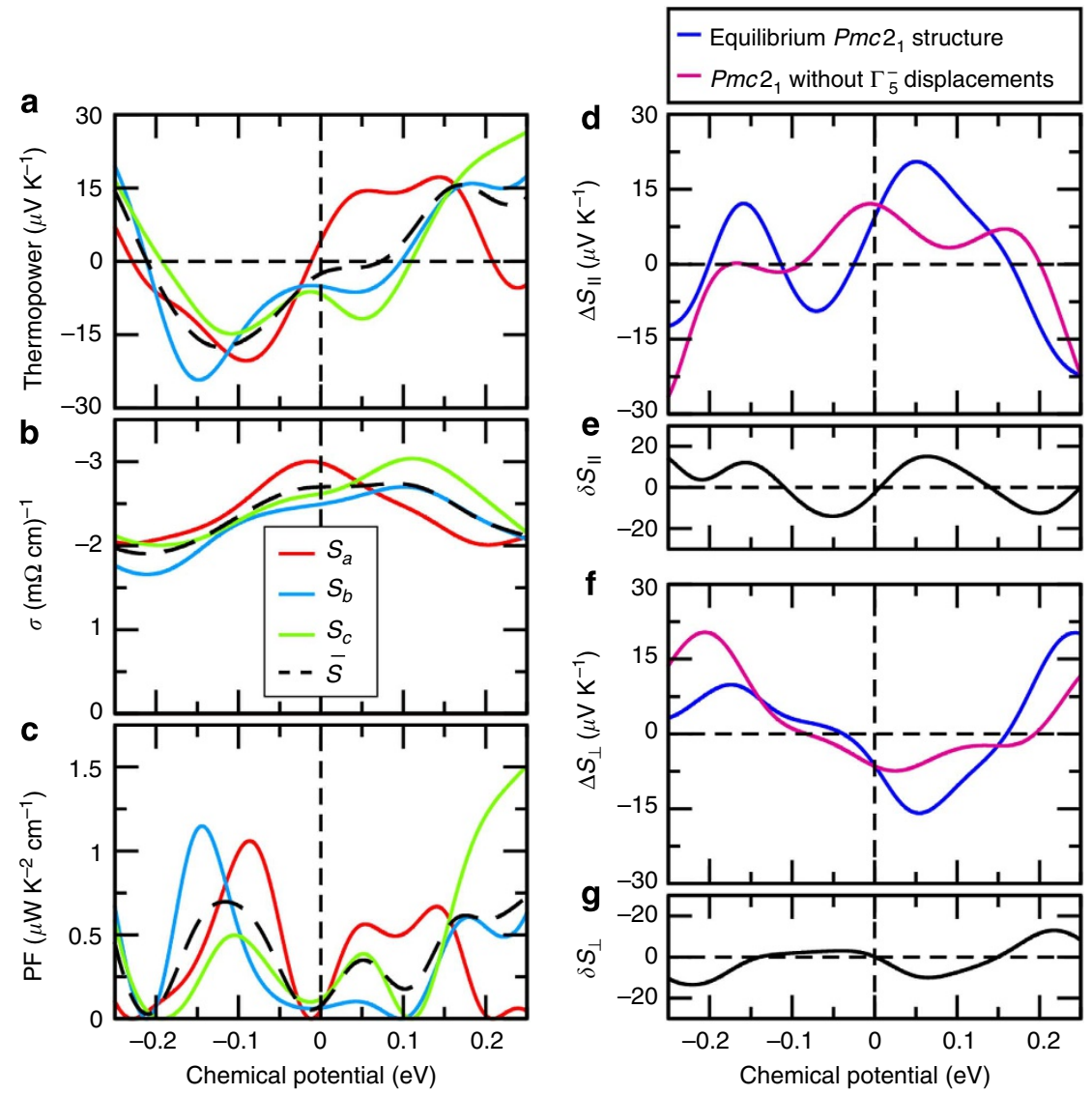

Figure 5 | Chemical potential-dependent properties and anisotropy at $\mathbf{3 0 0} \mathbf{K}$. ( $\mathrm{Sr}, \mathrm{Ca}$ ) $\mathrm{Ru}_{2} \mathrm{O}_{6}$ transport properties (left panels) as a function of the chemical potential $\mu$ : (a) thermopower $S_{1}(b)$ conductivity $\sigma$ and (c) the power factor PF. Red, blue and green lines are the transport properties along the $a, b$ and the polar $c$ axis, respectively. The dashed line indicates the total behaviour, given as an average of the three principle components. Anisotropy (right panels) along (d) the $a b$ plane $\left(\Delta S_{\|}\right)$with (blue) and without (magenta) polar displacements; the difference of the two curves is depicted in (e). (f) The anisotropy between the polar $c$ axis and the $a b$ plane $\left(\Delta S_{\perp}\right)$, and $(\mathbf{g})$ the difference between the two curves.

state structure exceeds that of the phase with the polar displacements removed.

Figure 6 shows the computed temperature-dependent thermopower $S$ as a function of chemical potential $\mu=0$ and $0.18 \mathrm{eV}$, which is selected because the power factor $\left(\sigma S^{2}\right)$ exhibits a local maximum that is due mainly to the Seebeck coefficient along the polar axis, $S_{c}$ (Fig. $5 c$ ). Due to identical lattice constants along the $b$ and $c$ axes in $(\mathrm{Sr}, \mathrm{Ca}) \mathrm{Ru}_{2} \mathrm{O}_{6}, S_{b}$ and $S_{c}$ have similar behaviour for $\mu=0 \mathrm{eV}$. The total thermopower, $\bar{S}$, ranges from $\sim 2.5$ to $-7 \mu \mathrm{V} \mathrm{K}^{-1}$ and exhibits parabolic behaviour with a maximum around $550 \mathrm{~K}$. The thermopower anisotropy along the polar $c$ axis, $\Delta S_{\perp}$, exhibits the same behaviour. At $\mu=0 \mathrm{eV}$, the thermopower anisotropy is relatively large $\left(\left|\Delta S_{\perp}\right| \sim 6.3 \mu \mathrm{V} \mathrm{K}^{-1}\right)$, reflected in the $300 \mathrm{~K}$ representation of the second-rank Seebeck tensor (inset) appearing as a hyperboloid with two sheets.

For $\mu=0.18 \mathrm{eV}$ the scenario is different. $\bar{S}$ has a quasi-linear behaviour from low to high temperature: $S_{a}, S_{b}$ and $S_{c}$ all become positive. Over the entire temperature range considered, the thermopower along the polar axis $S_{c}$ dominates. At $300 \mathrm{~K}$ (broken vertical line), the second-rank Seebeck tensor quadric surface becomes an elongated ellipsoid, flattened along the $c$ direction. Accordingly the thermopower anisotropy at $300 \mathrm{~K}$ becomes $\sim 5.8 \mu \mathrm{V} \mathrm{K}^{-1}$.

\section{Discussion}

The thermoelectric behaviour as a function of doping can be understood by examining the electronic band structures of the equilibrium $P m c 2_{1}$ structure with that of the hypothetical $(\mathrm{Sr}, \mathrm{Ca}) \mathrm{Ru}_{2} \mathrm{O}_{6}$ system with the polar atomic displacements removed (Fig. 7). Inspection of the electronic band structure along the lines of symmetry $X-S$ (corresponding to $b$ in real space) and $S-R$ (corresponding to the polar $c$ axis) reveals strongly hybridized $\mathrm{Ru} 4 d-\mathrm{O} 2 p$ bands dispersing from $-0.2 \mathrm{eV}$ to $0.3 \mathrm{eV}$, where the electron contribution dominates until $\sim 0.07 \mathrm{eV}$ above the Fermi level (Fig. 7a), explaining the sign change of $\bar{S}$ near $\mu \simeq 0.07 \mathrm{eV}$ in Fig. 5a.

In addition, the polar displacements largely modify the band structure along $S-R$ in the energy range $0.05-0.30 \mathrm{eV}$. In the absence of the polar mode, the band near $0.16 \mathrm{eV}$ (arrow in Fig. 7), which is derived from the oxygen $2 p$ states in the $\mathrm{CaO}$ and SrO planes, shifts to lower energy and broadens. In the equilibrium structure, this band along $S-R$ is higher in energy than the band which changes dispersion along $\Gamma-X$ (energy range $0.05-0.18 \mathrm{eV})$; however, when the polar displacements are removed, the oxygen-derived band along $S-R$ becomes lower than that of the band centre of mass along $\Gamma-X$. It is the dependence of these two bands on the amplitude of the polar atomic displacements that leads to the different behaviour appearing in Fig. 5f for $\mu=0.18 \mathrm{eV}$.

We find that the $300 \mathrm{~K}$ thermopower anisotropy of $(\mathrm{Sr}, \mathrm{Ca}) \mathrm{Ru}_{2} \mathrm{O}_{6}$ is close to that of centrosymmetric $\mathrm{YBa}_{2} \mathrm{Cu}_{3} \mathrm{O}_{7-\delta}$ (YBCO, $\left.\sim 7-10 \mu \mathrm{V} \mathrm{K}^{-1}\right)^{45,46}$, which exhibits strong thermal and electrical anisotropy owing to the two-dimensional (2D) crystal structure. The thermopower anisotropy in other metallic systems, for example, the hexagonal delafossite oxides $\mathrm{PdCoO}_{2}$ and 

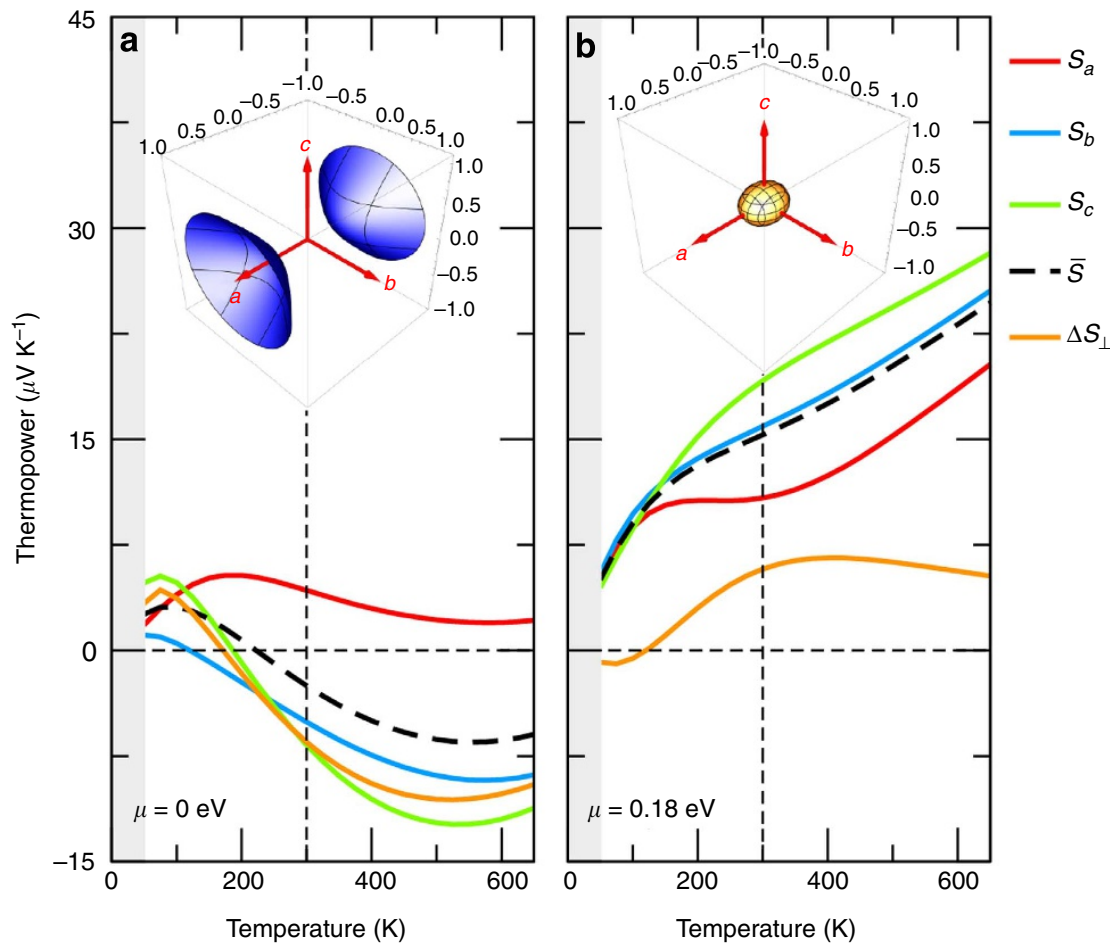

Figure 6 | Temperature-dependent thermopower in paramagnetic $(\mathbf{S r}, \mathbf{C a}) \mathbf{R} \mathbf{u}_{\mathbf{2}} \mathbf{O}_{\mathbf{6}}$. Temperature-dependent thermopower along the $a, b$ and $c$ axes at (a) $\mu=0 \mathrm{eV}$ and (b) $\mu=0.18 \mathrm{eV}$. The shaded area indicates the ferromagnetic phase below the calculated Curie temperature $T_{c} \sim 50 \mathrm{~K}$. The average thermopower is calculated as $\bar{S}=\left(S_{a}+S_{b}+S_{c}\right) / 3$. The insets depict the $300 \mathrm{~K}$ quadric surface representations of the second-rank Seebeck tensor, revealing enhanced anisotropy on doping along the polar axis.
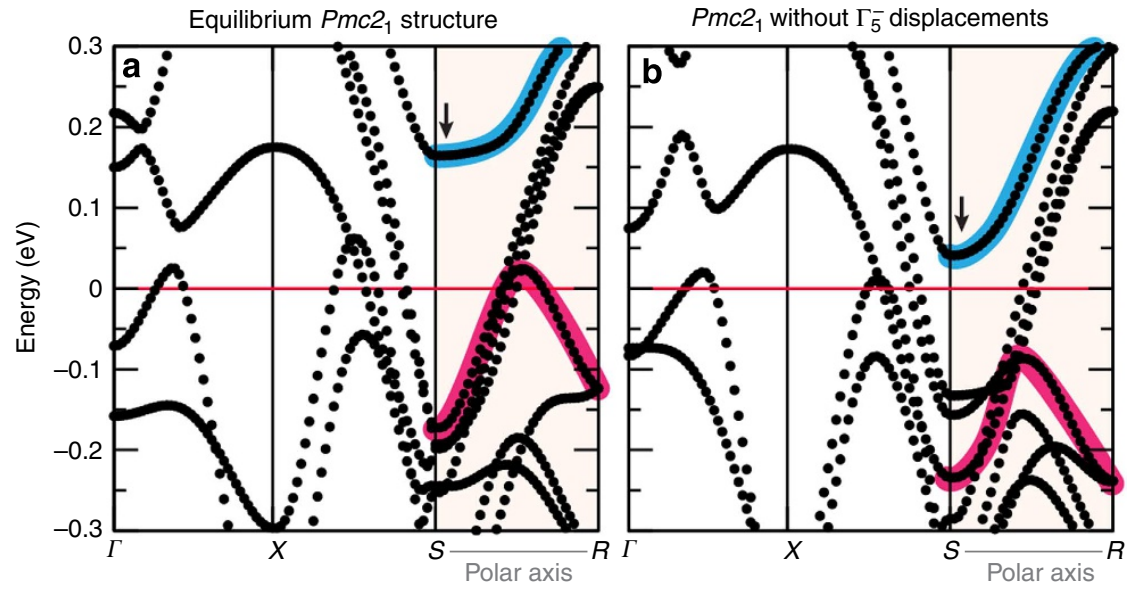

Figure 7 | Effect of polar displacements on the electronic structure. In the left (right) panel the bands structure for the non-magnetic layered $(\mathrm{Sr}, \mathrm{Ca}) \mathrm{Ru}_{2} \mathrm{O}_{6}$ (without $\Gamma_{5}^{-}$displacements). The highlighted bands are mainly affected by the polar displacements. The red (lower energy) bands are partially occupied in the equilibrium structure. The shaded path corresponds to a $k$-space trajectory parallel to the polar axis. Brillouin zone labels: $\Gamma$ ( 0,0 , $0), X(\pi / 2,0,0), S(\pi / 2, \pi / 2,0), R(\pi / 2, \pi / 2, \pi / 2)$.

$\mathrm{PtCoO}_{2}$ (ref. 47), also derive from the 2D layered topology of the system, and so present large Seebeck coefficients along the trigonal axis, that is, the axis which is most insulating. In contrast, the thermopower anisotropy in $(\mathrm{Sr}, \mathrm{Ca}) \mathrm{Ru}_{2} \mathrm{O}_{6}$ is not related to the dimensionality of the system, but rather to the existence of a polar axis along which the electrical conductivity is highest.

The peculiar thermopower anisotropy in $(\mathrm{Sr}, \mathrm{Ca}) \mathrm{Ru}_{2} \mathrm{O}_{6}$ dictates that the electric field resulting from an applied heat flux to the material will be non-collinear. This property is a fundamental feature for any anisotropic thermoelectric devices ${ }^{48}$. It enables the heat flux to be measured in a geometry perpendicular to the induced electrical current, specifically at locations where the temperatures are equal (see Supplementary Fig. 4 and Supplementary Note 1). Given the metallic conductivity of $(\mathrm{Sr}, \mathrm{Ca}) \mathrm{Ru}_{2} \mathrm{O}_{6}$ and that the relaxation time is small in comparison with that of semiconductors or insulators already finding use in thermoelectric devices, this polar NCSM would enable sensing on sub-nanosecond timescales (comparable to $\mathrm{YBCO}^{46}$ ). New applications of these oxide materials could be found in ultrafast thermoelectric devices ${ }^{46,48}$, where stability under extreme conditions, speed, and the ability to measure heat fluxes of high density are key requirements, for example, thermal (heat) radiation detectors. Moreover, because of the compatibility of perovskite oxides with Si-based CMOS technologies ${ }^{49}$, we 
anticipate that these designed NCSMs will more readily find integration and device development than previously identified materials with large anisotropic thermoelectric responses.

In summary, we designed a polar noncentrosymmetric ruthenate conductor and described how to eliminate the incompatibility between metallicity and acentricity in complex transition metal oxides. We articulated a paradigm for which new NCMS may be found, and showed that $(\mathrm{Sr}, \mathrm{Ca}) \mathrm{Ru}_{2} \mathrm{O}_{6}$ exhibits Seebeck coefficients with anisotropy derived from the polar structure. We believe that other polar metals could exhibit such anisotropic thermal properties; the important discriminating feature, however, is the $k$-space evolution of the electronic structure with the real space polar distortion. Broadly, such thermopower anisotropy may also be found in any NCSM, for example, in compounds which are chiral but non-polar, but continued studies are required to clarify the extent of the response. We hope this work motivates the synthesis of new materials and the discussion of new applications where highly anisotropic thermoelectric responses in metals can be leveraged.

\section{Methods}

$\boldsymbol{A} \boldsymbol{b}$ initio calculations and group theoretical analysis. We perform first-principles density functional calculations within the local spin density approximation, which is known to provide a reliable description of perovskite ruthenates ${ }^{35,39,50}$ as implemented in the Vienna $A b$ initio Simulation Package ${ }^{51,52}$. Calculations carried out with the Perdew-Burke-Ernzerhof generalized gradient approximation functional revised for solids ${ }^{53}$ provide essentially the same results (see Supplementary Table 6). We use the projector augmented wave method ${ }^{54}$ to treat the core and valence electrons using a $550 \mathrm{eV}$ plane wave expansion and the following electronic configurations: $4 s^{2} 4 p^{6} 5 s^{2}(\mathrm{Sr}), 3 p^{6} 4 s^{2}(\mathrm{Ca}), 4 p^{6} 5 s^{2} 4 d^{6}(\mathrm{Ru})$ and $2 s^{2} 2 p^{4}(\mathrm{O})$. A $7 \times 7 \times 5$ Monkhorst-Pack $k$-point mesh ${ }^{55}$ and Gaussian smearing ( $20 \mathrm{meV}$ width $)$ was used for the Brillouin zone (BZ) sampling and integrations.

For structure optimization, we relax the atomic positions to have forces less than $0.1 \mathrm{meV}^{-1}$. We impose as a constraint $b=c$ (note that in bulk ruthenates the deviations between the 'short' axes are small ${ }^{39}$ ) to simulate the situation under thin-film growth on a cubic substrate with a square lattice net. We search for the ground state structure by computing the phonon frequencies and eigenmodes of the high-symmetry centrosymmetric $(\mathrm{Sr}, \mathrm{Ca}) \mathrm{Ru}_{2} \mathrm{O}_{6}$ structure (space group $P 4 / \mathrm{mmm}$ ), and then systematically 'freeze-in' linear combinations of the unstable modes, performing structural optimization on these candidate low-symmetry structures until the global minimum is obtained. The group theoretical analysis was aided with the ISODISTORT ${ }^{56}$ and AMPLIMODES ${ }^{57,58}$ software.

Thermoelectric properties. For the calculation of the Seebeck coefficient and other transport properties, we use Boltzmann transport theory ${ }^{59,60}$ within the constant scattering time $(\tau)$ approximation as implemented in the boltztrap code ${ }^{61}$. We perform a non-self-consistent band-structure calculation with a much denser sampling of $27 \times 33 \times 33$ (4,046 $k$-points in the irreducible BZ; 29,403 $k$-points in the full BZ), using the equilibrium ground state structure. The Seebeck coefficients $S$ are calculated independently from $\tau$. The electrical conductivity, however, requires a known value of $\tau$, and so we introduce an empirical parameter. Here, the relaxation time value $\tau=0.23 \times 10^{-14} \mathrm{~s}$ is employed in all calculations, which we obtain from fitting the room-temperature conductivity ${ }^{62} \sigma \sim 2.7 \times 10^{5} \mathrm{~S} \mathrm{~m}^{-1}$ of solid solution $\mathrm{Sr}_{0.5} \mathrm{Ca}_{0.5} \mathrm{RuO}_{3}$. We believe this estimate to provide a meaningful approximation based on our excellent agreement with other physical properties of the solid solution reported in the main text.

The thermoelectric properties at room temperature are calculated using the zero-Kelvin polar structure based on the fact that the high-temperature structural transitions away from orthorhombic symmetry in bulk $\mathrm{CaRuO}_{3}$ and $\mathrm{SrRuO}_{3}$ do not occur until $1,550 \mathrm{~K}^{63}$ and $685 \mathrm{~K}$, respectively ${ }^{64}$. On the basis of our calculated magnetic Curie temperatures of $\sim 50 \mathrm{~K}$, we also compute all thermoelectric responses using a non-magnetic configuration.

\section{References}

1. Lines, M. E. \& Glass, A. M. Principles and Applications of Ferroelectrics and Related Materials (Clarendon, 1977).

2. Zhong, W., Vanderbilt, D. \& Rabe, K. M. Phase transitions in $\mathrm{BaTiO}_{3}$ from first principles. Phys. Rev. Lett. 73, 1861-1864 (1994).

3. Anderson, P. W. \& Blount, E. I. Symmetry considerations on martensitic transformations: "ferroelectric" metals? Phys. Rev. Lett. 14, 217-219 (1965)

4. Lawson, A. C. \& Zachariasen, W. H. Low temperature lattice transformation of $\mathrm{HfV}_{2}$. Phys. Lett. A 38, 1 (1972).

5. Mineev, V. P. \& Yoshioka, Y. Optical activity of noncentrosymmetric metals. Phys. Rev. B 81, 094525 (2010).
6. Edelstein, V. M. Features of light reflection off metals with destroyed mirror symmetry. Phys. Rev. B 83, 113109 (2011).

7. Edelstein, V. M. Magnetoelectric effect in polar superconductors. Phys. Rev. Lett. 75, 2004-2007 (1995)

8. Edelstein, V. M. Magnetoelectric effect in dirty superconductors with broken mirror symmetry. Phys. Rev. B 72, 172501 (2005).

9. Edelstein, V. M. The Ginzburg-Landau equation for superconductors of polar symmetry. J. Phys. Condens. Matter 8, 339-349 (1996).

10. Bauer, E. et al. Superconductivity in the complex metallic alloy $\beta-\mathrm{Al}_{3} \mathrm{Mg}_{2}$ Phys. Rev. B 76, 014528 (2007).

11. Bauer, E. et al. Unconventional superconducting phase in the weakly correlated noncentrosymmetric $\mathrm{Mo}_{3} \mathrm{Al}_{2} \mathrm{C}$ compound. Phys. Rev. B 82, 064511 (2010).

12. Poeppelmeier, K. R. et al. New family of planar cuprate superconductors: effect of nonmagnetic chains and planes. Phys. C Supercond. 185-189, 525-526 (1991).

13. Vaughey, J. T. et al. Synthesis and structure of a new family of cuprate superconductors: $\mathrm{LnSr}_{2} \mathrm{Cu}_{2} \mathrm{GaO}_{7}$. Chem. Mater. 3, 935-940 (1991).

14. Sergienko, I. A. et al. Metallic "ferroelectricity" in the Pyrochlore $\mathrm{Cd}_{2} \operatorname{Re}_{2} \mathrm{O}_{7}$. Phys. Rev. Lett. 92, 065501 (2004).

15. Shi, Y. et al. A ferroelectric-like structural transition in a metal. Nat. Mater. 12, 1024-1027 (2013).

16. Kolodiazhnyi, T., Tachibana, M., Kawaji, H., Hwang, J. \& Takayama-Muromachi, E. Persistence of ferroelectricity in $\mathrm{BaTiO}_{3}$ through the insulator-metal transition. Phys. Rev. Lett. 104, 147602 (2010).

17. Das, A. K. et al. Studies on temperature dependent semiconductor to metal transitions in $\mathrm{ZnO}$ thin films sparsely doped with Al. J. Appl. Phys. 112, 103706 (2012).

18. Cao, G., McCall, S., Shepard, M., Crow, J. E. \& Guertin, R. P. Thermal, magnetic, and transport properties of single-crystal $\mathrm{Sr}_{1-x} \mathrm{Ca}_{x} \mathrm{RuO}_{3}$ $(0 \lesssim x \lesssim 1.0)$. Phys. Rev. B 56, 321-329 (1997).

19. Wang, Y., Liu, X., Burton, J. D., Jaswal, S. S. \& Tsymbal, E. Y. Ferroelectric instability under screened coulomb interactions. Phys. Rev. Lett. 109, 247601 (2012).

20. Benedek, N. A. \& Fennie, C. J. Hybrid improper ferroelectricity: a mechanism for controllable polarization-magnetization coupling. Phys. Rev. Lett. 106, 107204 (2011).

21. Bousquet, E. et al. Improper ferroelectricity in perovskite oxide artificial superlattices. Nature 452, 732-736 (2008).

22. Howard, C. J. \& Stokes, H. T. Group-theoretical analysis of octahedral tilting in perovskites. Acta Crystallogr. B 54, 782-789 (1998).

23. King, G. \& Woodward, P. M. Cation ordering in perovskites. J. Mater. Chem. 20, 5785-5796 (2010).

24. Dachraoui, W. et al. Short-range layered a-site ordering in double perovskites NaLaBB'O6 (B = Mn, Fe; B' = Nb, Ta). Chem. Mater. 23, 2398-2406 (2011).

25. Zubko, P., Gariglio, S., Gabay, M., Ghosez, P. \& Triscone, J.-M. Interface physics in complex oxide heterostructures. Annu. Rev. Condens. Matter Phys. 2, 141-165 (2011).

26. Rondinelli, J. M. \& Fennie, C. J. Octahedral rotation-induced ferroelectricity in cation ordered perovskites. Adv. Mater. 24, 1961-1968 (2012).

27. Mulder, A. T., Benedek, N. A., Rondinelli, J. M. \& Fennie, C. J. Turning $\mathrm{ABO}_{3}$ anti-ferroelectrics into ferroelectrics: design rules for practical rotation-driven ferroelectricity in double perovskites and $\mathrm{A}_{3} \mathrm{~B}_{2} \mathrm{O}_{7}$ Ruddlesden-Popper compounds. Adv. Funct. Mater. 23, 4810-4820 (2013).

28. Woodward, P. M. Octahedral tilting in perovskites. II. Structure stabilizing forces. Acta Crystallogr. B 53, 44-66 (1997).

29. Kunz, Martin. \& Brown, I. David. Out-of-center distortions around octahedrally coordinated $\mathrm{d}^{0}$ transition metals. J. Solid State Chem. 115, 395-406 (1995).

30. Kobayashi, H., Nagata, M., Kanno, R. \& Kawamoto, Y. Structural characterization of the orthorhombic perovskites: $\left[\mathrm{ARuO}_{3}(\mathrm{~A}=\mathrm{Ca}, \mathrm{Sr}, \mathrm{La}, \mathrm{Pr})\right]$. Mater. Res. Bull. 29, 1271-1280 (1994).

31. Lampis, N., Franchini, C., Satta, G., Geddo-Lehmann, A. \& Massidda, S. Electronic structure of $\mathrm{PbFe}_{1 / 2} \mathrm{Ta}_{1 / 2} \mathrm{O}_{3}$ : crystallographic ordering and magnetic properties. Phys. Rev. B 69, 064412 (2004).

32. Anderson, P. W. New approach to the theory of superexchange interactions. Phys. Rev. 115, 2-13 (1959).

33. Wu, H., Hu, Z., Khomskii, D. I. \& Tjeng, L. H. Insulating state and the importance of the spin-orbit coupling in $\mathrm{Ca}_{3} \mathrm{CoRhO}_{6}$. Phys. Rev. B 75, 245118 (2007).

34. Maitra, T. \& Valenti, R. Orbital Order in $\mathrm{ZnV}_{2} \mathrm{O}_{4}$. Phys. Rev. Lett. 99, 126401 (2007).

35. Rondinelli, J. M., Caffrey, N. M., Sanvito, S. \& Spaldin, N. A. Electronic properties of bulk and thin film $\mathrm{SrRuO}_{3}$ : search for the metal-insulator transition. Phys. Rev. B 78, 155107 (2008).

36. Aroyo, M. I., Kirov, A., Capillas, C., Perez-Mato, J. M. \& Wondratschek, H. Bilbao Crystallographic Server. II. Representations of crystallographic point groups and space groups. Acta Crystallogr. A 62, 115-128 (2006). 
37. Orobengoa, D., Capillas, C., Aroyo, M. I. \& Perez-Mato, J. M. AMPLIMODES: symmetry-mode analysis on the Bilbao Crystallographic Server. J. Appl. Crystallogr. 42, 820-833 (2009).

38. Lee, J. H. \& Rabe, K. M. Epitaxial-strain-induced multiferroicity in $\mathrm{SrMnO}_{3}$ from first principles. Phys. Rev. Lett. 104, 207204 (2010).

39. Zayak, A. T., Huang, X., Neaton, J. B. \& Rabe, K. M. Manipulating magnetic properties of $\mathrm{SrRuO}_{3}$ and $\mathrm{CaRuO}_{3}$ with epitaxial and uniaxial strains. Phys. Rev. B 77, 214410 (2008).

40. Garcia-Fernandez, P., Verissimo-Alves, M., Bilc, D. I., Ghosez, P. \& Junquera, J. First-principles modeling of the thermoelectric properties of $\mathrm{SrTiO}_{3} / \mathrm{SrRuO}_{3}$ superlattices. Phys. Rev. B 86, 085305 (2012).

41. Lee, S., Bock, J. A., Trolier-McKinstry, S. \& Randall, C. A. Ferroelectric-thermoelectricity and mott transition of ferroelectric oxides with high electronic conductivity. J. Eur. Ceram. Soc. 32, 3971-3988 (2012).

42. Xi, T. G., Fei, Y., Choy, C. L., Leung, W. P. \& Shao, C. F. Specific heat and thermal diffusivity of strontium barium niobate $\left(\mathrm{Sr}_{1-{ }_{x}} \mathrm{Ba}_{\mathrm{x}} \mathrm{Nb}_{2} \mathrm{O}_{6}\right)$ single crystals. J. Eur. Ceram. Soc. 71, 170-173 (1992).

43. Kolodiazhnyi, T. et al. Thermoelectric power, Hall effect, and mobility of n-type $\mathrm{BaTiO}_{3}$. Phys. Rev. B 68, 085205 (2003).

44. Snyder, G. J. \& Toberer, E. S. Complex thermoelectric materials. Nat. Mater. 7, 105-114 (2008).

45. Kaiser, A. B. \& Uher, C. in Studies of High Temperature Superconductors Vol. 7 (ed. Narlikar, A. V.) 353 (Nova Science, 1991).

46. Prettl, W., Zeuner, S. \& Lengfellner, H. Fast thermoelectric response of normal state $\mathrm{YBa}_{2} \mathrm{Cu}_{3} \mathrm{O}_{7-\delta}$ films. Appl. Phys. Lett. 66, 1833-1835 (1995).

47. Ong, K. P., Singh, D. J. \& Wu, P. Unusual transport and strongly anisotropic thermopower in $\mathrm{PtCoO}_{2}$ and $\mathrm{PdCoO}_{2}$. Phys. Rev. Lett. 104, 176601 (2010).

48. Burkov, A. T., Heinrich, A. \& Vedernikov, M. V. Anisotropic thermoelectric materials, properties and applications. AIP Conf. Proc. 316, 76-80 (1994).

49. Warusawithana, M. P. et al. A ferroelectric oxide made directly on silicon,". Science 324, 367-370 (2009).

50. Zayak, A. T., Huang, X., Neaton, J. B. \& Rabe, K. M. Structural, electronic, and magnetic properties of SrRuO3 under epitaxial strain. Phys. Rev. B 74, 094104 (2006).

51. Kresse, G. \& Furthmuller, J. Efficient iterative schemes for ab initio total-energy calculations using a plane-wave basis set. Phys. Rev. B 54, 11169-11186 (1996).

52. Kresse, G. \& Joubert, D. From ultrasoft pseudopotentials to the projector augmented-wave method. Phys. Rev. B 59, 1758-1775 (1999).

53. Perdew, J. P. et al. Restoring the density-gradient expansion for exchange in solids and surfaces. Phys. Rev. Lett. 100, 136406 (2008).

54. Blochl, P. E. Projector augmented-wave method. Phys. Rev. B 50, 17953-17979 (1994).

55. Monkhorst, H. J. \& Pack, J. D. Special points for Brillouin-zone integrations Phys. Rev. B 13, 5188-5192 (1976).

56. Campbell, B. J., Stokes, H. T., Tanner, D. E. \& Hatch, D. M. ISODISPLACE a web-based tool for exploring structural distortions. J. Appl. Crystallogr. 39, 607-614 (2006)
57. Orobengoa, D., Capillas, C., Aroyo, M. I. \& Perez-Mato, J. M. AMPLIMODES: symmetry-mode analysis on the Bilbao Crystallographic Server. J. Appl. Crystallogr. 42, 820-833 (2009).

58. Perez-Mato, J. M., Orobengoa, D. \& Aroyo, M. I. Mode crystallography of distorted structures. Acta Crystallogr. A 66, 558-590 (2010).

59. Ashcroft, N. W. \& Mermin, N. D. Solid State Physics (Thomson Learning, Inc., 1976).

60. Ziman, J. M. Principles of the Theory of Solids (University Press, 1972).

61. Madsen, G. K. H. \& Singh, D. J. Boltztrap. a code for calculating band-structure dependent quantities. Comput. Phys. Commun. 175, 67-71 (2006).

62. Keawprak, N., Tu, R. \& Goto, T. Thermoelectric properties of $\mathrm{Ca}_{1-} \mathrm{Sr}_{\mathrm{x}} \mathrm{RuO}_{3}$ compounds prepared by spark plasma sintering. J. Alloys Compd. 523, 182-187 (2012).

63. Vashook, V. et al. Structural stability of conducting oxide $\mathrm{CaRuO}_{3}$ at high temperatures. Appl. Phys. Lett. 90, 251913 (2007).

64. Kennedy, B. J., Hunter, B. A. \& Hester, J. R. Synchrotron X-ray diffraction reexamination of the sequence of high-temperature phases in $\mathrm{SrRuO}_{3}$. Phys Rev. B 65, 224103 (2002)

65. Halasyamani, P. S. \& Poeppelmeier, K. R. Non-centrosymmetric oxides. Chem. Mater. 10, 2753-2769 (1998).

\section{Acknowledgements}

This work was supported by the Army Research Office under grant no. W911NF-12-1-0133. We acknowledge the High Performance Computing Modernization Program (HPCMP) of the DOD for providing computational resources that have contributed to the research results reported herein. We thank K.R. Poepplemeier and C.J. Fennie for insightful discussions.

\section{Author contributions}

The study was planned, calculations carried out and the manuscript prepared by D.P. and J.M.R. Both authors discussed the results, wrote and commented on the manuscript.

\section{Additional information}

Supplementary Information accompanies this paper at http://www.nature.com/ naturecommunications

Competing financial interests: The authors declare no competing financial interests.

Reprints and permission information is available online at http://npg.nature.com/ reprintsandpermissions/

How to cite this article: Puggioni, D. et al. Designing a robustly metallic noncenstrosymmetric ruthenate oxide with large thermopower anisotropy Nat. Commun. 5:3432 doi: 10.1038/ncomms4432 (2014). 